\title{
Enhanced vision flight deck technology for commercial aircraft low-visibility surface operations
}

\author{
Jarvis (Trey) J. Arthur, III ${ }^{a}$, R. Michael Norman ${ }^{b}$, Lynda J. Kramer ${ }^{a}$, Lawrence J. \\ Prinzel, III ${ }^{a}$, Kyle K. E. Ellis ${ }^{a}$, Stephanie J. Harrison ${ }^{c}$ and J. Ray Comstock ${ }^{a}$ \\ ${ }^{a}$ NASA Langley Research Center, Hampton, VA \\ ${ }^{b}$ Boeing Research and Technology, Hampton, VA \\ ${ }^{c}$ Virginia Polytechnic Institute and State University, Blacksburg, VA
}

\begin{abstract}
NASA Langley Research Center and the FAA collaborated in an effort to evaluate the effect of Enhanced Vision (EV) technology display in a commercial flight deck during low visibility surface operations. Surface operations were simulated at the Memphis, TN (FAA identifier: KMEM) airfield during nighttime with 500 Runway Visual Range (RVR) in a high-fidelity, full-motion simulator. Ten commercial airline flight crews evaluated the efficacy of various EV display locations and parallax and minification effects. The research paper discusses qualitative and quantitative results of the simulation experiment, including the effect of EV display placement on visual attention, as measured by the use of non-obtrusive oculometry and pilot mental workload. The results demonstrated the potential of EV technology to enhance situation awareness which is dependent on the ease of access and location of the displays. Implications and future directions are discussed.
\end{abstract}

Keywords: Enhanced Flight Vision Systems, Enhanced Vision, NextGen, Equivalent Visual Operations, Flight Simulation

\section{INTRODUCTION}

Research and experience has shown that reduced operational tempos and delays in current-day surface operations due to low visibility conditions contribute significantly, and are growing in their contribution, to National Airspace System (NAS) delays. To ensure the continuation of safe ground operations during low visibility-conditions, pilots must maintain awareness of: 1) their geographic position (i.e., location on the airport); 2) their cleared navigation routing; 3 ) the surface types and associated indications (e.g., runways, taxiways, maneuvering/non-maneuvering areas, hold lines, etc.) and, 4) their position with respect to other aircraft, vehicles, and obstacles/objects - all while keeping the aircraft on the pavement (i.e., maintaining path control).

Under an Inter-agency Agreement, the NASA Langley Research Center (LaRC) and the FAA are conducting collaborative research activities to ensure effective technology development and implementation of design standards for the Next Generation Air Transportation System (NextGen). As part of this agreement, research is being conducted to assess the introduction and use of Enhanced Vision (EV) and Enhanced Flight Vision Systems (EFVS) advanced cockpit vision technologies for Low Visibility Operations/Surface Movement Guidance and Control System (LVO/SMGCS).

\subsection{Surface Movement Guidance and Control System}

As counter-measures to potential loss-of-safety margins in low visibility conditions, the FAA has established regulations, standards, and supporting advisory material in the development of Surface Movement Guidance and Control System (SMGCS) requirements for U.S. airports where scheduled air carriers are authorized to conduct operations when the visibility is less than 1,200 feet Runway Visual Range (RVR). A SMGCS plan facilitates the safe movement of aircraft and vehicles on the airport by establishing rigorous control procedures and requiring enhanced visual aids. SMGCS involves surveillance, routing, guidance, and control for controllers,

Further author information: Jarvis (Trey) Arthur, E-mail: Trey.Arthur@nasa.gov, Telephone: 1-757-864-6609 
pilots, vehicle drivers, marshallers, emergency services, airport authorities, regulatory authorities, and security services. These plans address the information needs and define the roles and responsibilities of both of the Air Navigation Services Provider and to each aircraft and vehicle operating in the movement area.

Key enabling elements of SMGCS are enhanced visual aids (AC150/5340-30F) consisting of lights, markings, and signage on the airport designed to provide visual cues to pilots and vehicle operators sufficient to maintain their situation awareness and to ensure the continuation of safe, efficient ground operations. These enhanced visual aids establish mechanisms by which surveillance, routing, guidance, and control of surface operations in low visibility conditions may be achieved.

\subsection{Enhanced Vision Systems for commercial operations}

The Vehicle Systems and Safety Technologies (VSST) project, under NASA's Aviation Safety Program, is conducting research, development, test and evaluation of crew-vehicle interface technologies to increase the pilots' ability to avoid, detect, and recover from adverse events that could otherwise result in accidents/incidents. One goal is to proactively overcome aircraft safety barriers that might otherwise constrain the full realization of the NextGen. Part of this research effort involves the use of synthetic and enhanced vision systems and advanced display media as enabling crew-vehicle interface technologies to meet these safety challenges.

$\mathrm{EV}$ is an electronic means to provide a display of the external scene topography (the natural or man-made features of a place or region especially in a way to show their relative positions and elevation) through the use of an imaging sensor, such as a Forward Looking Infrared (FLIR) or millimeter wave radar. Development of applications of EV technology for commercial, business, and GA aircraft was energized in January $2004^{1}$ when Title 14 of the US Code of Federal Regulations (CFR) $§ 91.175$ was amended such that operators conducting straight-in instrument approach procedures (in other than Category II or Category III operations) could then operate below the published Decision Altitude (DA)/Decision Height (DH) or Minimum Descent Altitude (MDA) when using an approved EFVS. An EFVS, in this application, is an integrated conformal display of EV and symbology shown on the pilot's Head-Up Display (HUD). In most atmospheric conditions, especially when natural visibility is reduced due to night, smoke, or haze, the EV provides a visibility improvement over natural vision and it can be logically concluded that improvements in situation awareness (awareness of geographic position, of positioning on the runways and taxiways, and of objects, traffic, and other vehicles) are derived. This information may enable the flight crew (pilot) to more safely operate on the surface, including taxi, parking, and gate operations, or to conduct these operations in weather and visibility conditions for which this would normally be precluded. Such a goal is supported by the FAA's mid-term vision for NextGen. ${ }^{2}$ An operational goal may be to utilize flight deck equipage (i.e., EV) in lieu of LVO/SMGCS infrastructure requirements (e.g., enhanced visual aids).

As with any new technology, the integration within the cockpit must be carefully considered. The considerations include, for example, the EV display location and the associated cognitive and visual workload and attention in utilizing this information. Further, the integration must also consider how the pilot will fuse this information with the disparate other sources of navigation information, positional awareness, and traffic awareness available inside and outside the cockpit. ${ }^{3}$ This integration must consider the different frames of references, fields-of-regard, and display minification factors for each information source. The EV information itself may be displayed in an ego-centric or exo-centric format, shown in a head-up or head-down location with various fields-of-regard or minimization factor. (Note that an EFVS application requires a conformal, ego-centric EV display on the pilot's HUD). The process of information fusion must span the angular separation between the paper or electronic charts, the out-the-window information, flight-critical/task-critical instrumentation, and the EV display.

The present research is motivated by the need to quantify the influence of $\mathrm{EV}$ operations within the flight deck during surface operations, and identify if operational credit might be possible whereby an EV-approved and equipped aircraft could operate in prevailing visibilities lower than currently approved. Consideration of the field-of-regard/field-of-view, the viewing perspective (ego-centric vs. exo-centric), and the spatial location of the information were assumed to be critical factors. 


\section{EXPERIMENT}

The objective of the research presented in this paper was to evaluate the use of EV during LVO/SMGCS and assess the impact of different EV flight deck design considerations; specifically, EV display location, EV image minification, and EV sensor (camera) parallax.

\subsection{Simulation facility}

Simulation research was conducted in the NASA LaRC Research Flight Deck (RFD) motion based simulator (Fig. 1). The RFD was configured to mimic the instrument panel of current state-of-the-art commercial transport aircraft, with four 10.5" vertical by 13.25" horizontal, 1280x1024 pixel resolution, color displays tiled across the instrument panel. Also, the RFD included a mode control panel, Flight Management System (FMS), control display units, and hydraulic-actuated side-stick control inceptors. A collimated Out-the-Window (OTW) scene provided approximately $200^{\circ}$ horizontal by $40^{\circ}$ vertical field-of-view (FOV) at 26 pixels per degree.
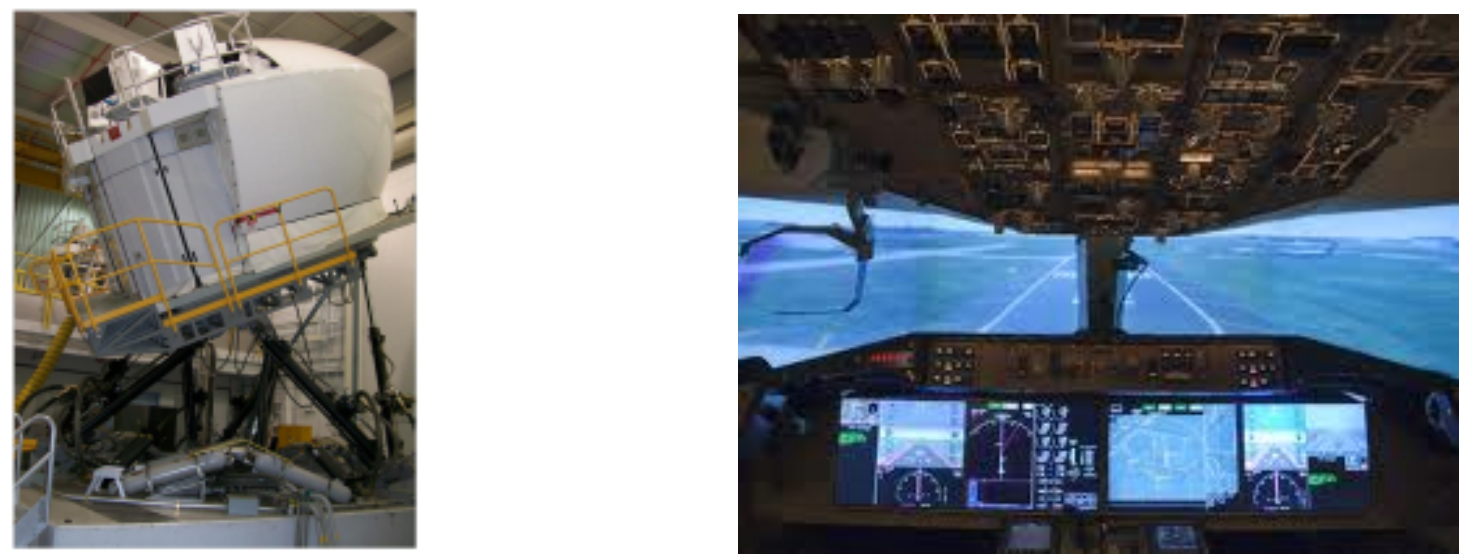

Figure 1. The Research Flight Deck motion simulator at NASA Langley Research Center.

Appropriate pre-recorded Airport-Terminal Information Service (ATIS) messages were presented (aurally) providing information on the environmental conditions and airport status (e.g., SMGCS operations in use) for each scenario.

The test facility had a navigation and communication facilities simulation that replicated realistic voice communication during approach, taxi-out, and taxi-in scenarios. The communications included pre-recorded voice communications to stage most of the scenarios and create realistic "party-line' chatter to promote realism. A researcher also provided simulated Air Traffic Control (ATC) directives and information to augment the pre-recorded ATC audio, as well as intervention when required (i.e., when a crew queried ATC with questions regarding a clearance).

\subsection{Enhanced vision simulation}

The EV was simulated as a combined short-wave, mid-wave ( 1.0 to 5.0 micron) FLIR sensor. The simulated sensor aperture (normal parallax condition) was placed $5.25 \mathrm{ft}$ below the pilot design eye point reference (DERP), $1.5 \mathrm{ft}$ to the right, and $6.5 \mathrm{ft}$ forward, simulating an aircraft "chin" installation. The simulated camera was aligned with the HUD, so any image shift between the FLIR displayed on the HUD and the OTW was due only to installation parallax. The image shift (i.e., error) due to camera parallax for this case was half of the maximum error allowable for an EFVS in accordance with RTCA DO- $315,{ }^{4}$ equating to a 2.5 mrad image offset of a point located at a $2000 \mathrm{ft}$ distance. A large parallax error condition was also simulated which moved the FLIR sensor location to a point $10.5 \mathrm{ft}$ below the DERP (lateral and longitudinal locations remained the same as before), equating to an apparent image shift of approximately $5 \mathrm{mrad}$ for a point at $2000 \mathrm{ft}$ distance, and equivalent to the maximum allowable error in accordance with RTCA DO-315. 


\subsection{EV display locations}

The location of the EV information in the cockpit was varied:

- EV was displayed on the HUD, assuming that the display was used as an EFVS. When other configurations were tested, the HUD was stowed. The HUD was collimated (focused at approximately optical infinity) and subtends $30^{\circ} \mathrm{H}$ by $24^{\circ} \mathrm{V}$ FOV.

- A 5.6 inch diagonal LCD was placed above the glare shield on the center post of the forward window ("Center Auxiliary Display (AD)").

- Two additional ADs with a diagonal of 8 inches were placed at $60^{\circ}$ and $90^{\circ}$ outboard from the longitudinal axis (" $60^{\circ} \mathrm{AD}$ " and " $90^{\circ} \mathrm{AD}$ "). During a data trial, only one of these (or none) ADs were turned-on at a given location.

- A 6 inch diagonal EV image in the upper left quadrant of the Pilot Monitoring (PM)'s Navigational Display (ND).

\subsection{Evaluation pilots}

Ten commercial flight crews from various US airlines participated in the experiment. The pilots were paired based upon their current employer to try to minimize intercrew conflicts in Standard Operating Procedures and Crew Resource Management procedures. Each pilot held an Airline Transport Pilot rating with at least 100 hours of HUD experience and had experience with EV.

\subsection{Flight crew training}

The Evaluation Pilots (EPs) were given a 45-minute classroom briefing to explain the display concepts and the evaluation tasks for the experiment. After the briefing, a 30-minute training session was conducted to familiarize the EPs with the RFD simulator. Following this training, 4 hours of data collection was conducted. At the end of the day, a post-test interview was conducted to solicit the crew's comments on the experiment. The total duty time for an EP was approximately 8 hours.

\subsection{Methodology}

The experimental design was a 5 (EV display location) by 2 (image scale/field-of-regard) by 2 (size of parallax error) partially factorial within-subjects design. In addition, a baseline condition (no EV/EFVS) was also tested to generate comparative performance data.

The selected configurations are shown in Table 1. Two EV sensor FOVs were simulated. As indicated in Table 1, this created 2 field-of-regard (FOR) (i.e., the angular extent of the external world represented on the display) conditions for testing. The "Normal" FOR corresponds to the sensor FOV which created a non-minified HUD EV display (the EV image FOR is approximately the same as the HUD). In other words, the EV image on the HUD was conformal in that it overlaid the real-world. This same sensor FOV was used on each of the other "Normal" FOR display concepts. The term "Minified" corresponds to when the sensor FOV was doubled. This caused a $2 \mathrm{x}$ minification factor (i.e., the ratio of the EV sensor FOV to the display FOV is doubled). As the camera sensor cannot be mounted at the pilot's eye, there will be inherent parallax in the EV image especially noticeable on the HUD. The terms "Small" and "Large" correspond to the degree of parallax in the EV display, effectively yielding 2.5 and 5.0 mrads of parallax error at a range of 2000 feet.

In addition to the conditions shown in Table 1, two additional run conditions were conducted: a baseline condition (no Enhanced Vision System (EVS)) and an off-nominal event. The single off-nominal scenario involved the detection of a baggage cart, positioned to be visible in the EV image, but not readily OTW. The off-nominal scenario was conducted with two EV locations (HUD and Center AD), thereby, investigating issues associated with different head-up positions of EV for the Pilot Flying (PF) and head-up versus head-down for the PM. The off-nominal scenario was unexpected by the flight crew and was used to evaluate latent safety issues associated with SMGCS operations. 


\begin{tabular}{cc|ccccc} 
Field-of-regard & Parallax & HUD & Center AD & $60^{\circ}$ AD & $90^{\circ}$ AD & MFD \\
\hline Normal (N) & Small & $\checkmark$ & $\checkmark$ & $\checkmark$ & $\checkmark$ & $\checkmark$ \\
Normal (N) & Large & $\checkmark$ & $\checkmark$ & $\checkmark$ & $\checkmark$ & $\checkmark$ \\
Minified (M) & Small & & $\checkmark$ & $\checkmark$ & $\checkmark$ & $\checkmark$ \\
Minified (M) & Large & $\checkmark$ & $\checkmark$ & $\checkmark$ & $\checkmark$ \\
\hline \multicolumn{7}{r}{ Table 1. Experiment matrix showing 18 nominal experiment conditions. }
\end{tabular}

\subsection{Evaluation task}

Simulated operations were conducted at the Memphis International Airport, TN (FAA identifier: KMEM). The simulated weather conditions were $500 \mathrm{ft}$ RVR at night (full dark conditions). The EV (i.e., FLIR) simulation was calibrated to show topographical objects within a range of approximately $600 \mathrm{ft}$ and light sources within a range of approximately $1000 \mathrm{ft}$. A terrain database was used for the KMEM area, which included all airport taxiways, runways, SMGCS visual aids and markings, prominent airport buildings, obstructions, signs, and airport terrain and cultural features. The simulator also used the appropriate database information to emulate the accurate location and appropriate radio frequencies of navigation aids, to coincide with published charts.

Crews were provided with KMEM LVO/SMGCS paper charts for surface operations. All expected procedures and appropriate protocols were briefed prior to the test for each crew, and training was provided to familiarize crews with operational procedures prior to data collection. Paper checklists appropriate to the B-757 were supplied and used by the crews.

Prior to each data run, crews were briefed on their position on the airport or in the air on approach. For departure scenarios, crews tuned the radio and listened to an an automated ATIS. Crews then called the ground controller to receive taxi instructions. If the PM failed to correctly read-back the proper taxi instructions, the taxi clearance was read to the crew until a correct read back occurred. Departures scenarios ended when ownship reached the hold short line for the take off runway.

For approach scenarios, crews were briefed they were starting at $1000 \mathrm{ft}$ height above terrain and the approach runway. Crews were then briefed on the weather conditions and allowed to conduct any briefings or checklist before the data trial began. Once crews contacted the tower, crews were given a landing clearance with a high speed turn-off, if feasible. Once cleared of the runway, crews were instructed to switch to ground frequency and get taxi instructions.

Post-run questionnaires were given to both EPs after each scenario, and consisted of a 1) NASA Task Load Index (TLX) workload rating, ${ }^{5}$ 2) a Situational Awareness Rating Technique (SART) form, ${ }^{6}$ and 3) questions addressing crew interaction, operational effectiveness, and EV usability. This questionnaires were given immediately after the end of each data trial.

Crews were given a Simulation Sickness Questionnaire (SSQ) ${ }^{7}$ throughout the day. The SSQ is a list of 16 symptoms (general discomfort, fatigue, nausea, etc) which the crews were asked if they were experiencing at that moment. If they did experience a symptom, they were asked to rate the severity of the symptom as slight, moderate or severe. A total of six SSQs were administered with one SSQ given at the beginning of the day and one SSQ given at the end of the day. The four remaining SSQs were given at regular intervals during data collection.

\subsection{Displays}

The head-down displays replicated a glass flight deck configuration as shown in Fig. 2.

\section{RESULTS}

Quantitative (i.e., aircraft state, navigational, systems interaction, eye tracking) data as well as qualitative (i.e., survey, questionnaire, workload and situation awareness metrics, pilot opinion) measures were recorded and used in a detailed data analysis to answer the purpose of the research. 

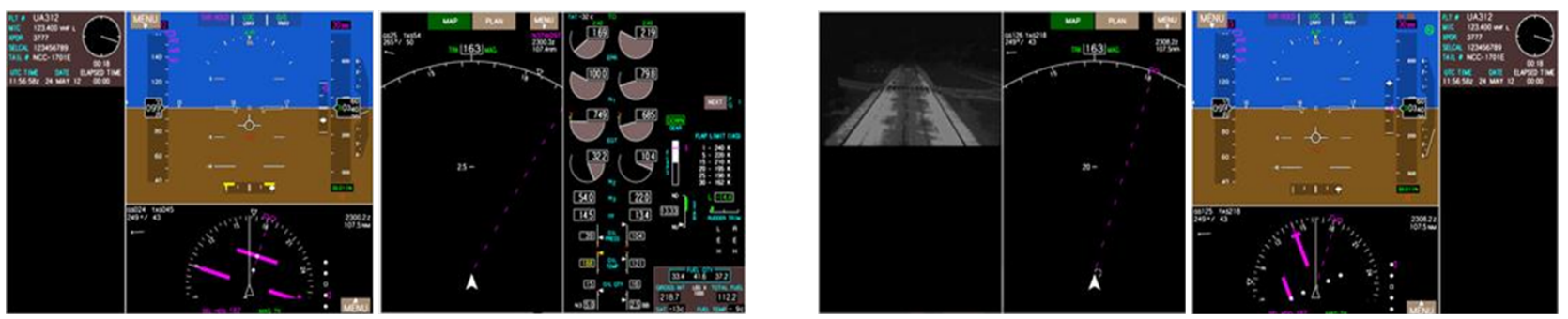

Figure 2. The PF head-down displays (left) and the PM head-down displays (right). The MFD (EV repeater) is shown in the upper left of the PM ND.

\subsection{Effects of display condition}

The 5 display conditions were: 1) HUD; 2) Center AD; 3) 60 AD; 4) 90 AD; and, 5) Multi-Function Display (MFD).

\subsubsection{Average taxi speed}

Average taxi speeds were calculated as the total distance traveled, divided by the elapsed time. For arrival scenarios, averaging started when ownship was below 30 knots ground speed on rollout and continued until the end of each run. For departure scenarios, averaging started when first above 1.0 knots ground speed, and continued until the end of each run.

An Analysis of Variance (ANOVA) was conducted on the average taxi speed (measured in knots) dependent variable for display condition (19 display conditions). Levenes test for equality of variances was met for the present analysis. Display condition was not significant, $F(18,161)=0.303, p=0.989$, for average taxi speed. The overall mean taxi speed was 11.4 knots with a standard deviation of 2.4 knots.

\subsubsection{Head up time, pilot flying}

Oculometers provided eye and head position and pointing vectors for each crew throughout the runs. An ANOVA was conducted on the PF head-up time percentage dependent variable for display condition (19 display conditions). Levenes test for equality of variances was met for the present analysis. Display condition was not significant, $F(18,161)=1.013, p=0.449$, for PF head-up percentage time. Operationally, the presence of $\mathrm{EV}$ and where it was displayed did not affect the PF head-up percentage time when compared to conventional displays without EV information. The overall mean PF head-up percentage time was $66.2 \%$ with a standard deviation of $19.8 \%$.

\subsubsection{Head-up time, pilot monitoring}

Levene's test for equality of variances was not met for the PM head-up percentage time. Logarithmic-transformed head-up time percentage data met Levene's test for equality of variances and was used in an ANOVA on the PM head-up time percentage data $^{8}$ for display condition (19 display conditions). When means of the logarithmic PM head-up percentage time data are transformed back to original units, the mean reported is actually the geometric mean of the head-up percentage time and that is what is reported. Display condition was not significant, $F(18,157)=1.507, p=0.094$, for PM head-up percentage time. Operationally, the presence of EV and where it was displayed did not affect the PM head-up percentage time when compared to conventional displays without EV information. The overall (geometric) mean PM head-up percentage time was 53.0\%.

\subsubsection{Situation awareness}

A SART was also administered after each run. The SART incorporated three dominant components: demand on the pilot's resources, supply of resources, and understanding of the situation. Pilots rated their perception of the impact of these components using a 7-point Likert scale.

An ANOVA was conducted on the SART dependent variable for display condition. Mauchly's Test of Sphericity was not significant $(p=0.056)$. The omnibus F-test revealed a statistically significant difference for display 
condition, $F(18,342)=2.626, p<0.001$. However, the pair-wise comparison analyses with Bonferroni correction failed to reveal any significant effects across display conditions $(p>0.05)$.

A planned comparison was conducted contrasting each of the display conditions (18 conditions) to the baseline condition (No HUD, No EV). This analysis evinced some significant contrasts. The following display conditions were rated significantly worse $(p<0.05)$ than the baseline condition $(\mathrm{SART}=7.80)$ :

1. $90^{\circ} \mathrm{AD}$, Normal FOR (N), Small Parallax, $F(1,19)=4.701, p=0.043$

2. $90^{\circ} \mathrm{AD}$, Normal FOR (N), Large Parallax, $F(1,19)=7.325, p=0.014$

3. $90^{\circ} \mathrm{AD}$, Minified FOR (M), Large Parallax, $F(1,19)=4.750, p=0.042$

4. $90^{\circ} \mathrm{AD}$, Minified FOR (M), Small Parallax, $F(1,19)=6.577, p=0.019$

This result indicates that pilots rated the $90^{\circ} \mathrm{AD}$ location as significantly worse for situation awareness compared to the baseline condition. Pilot comments indicate that this reduction in situational awareness (SA) was largely due to its location outside the primary or secondary field-of-view compared to the baseline condition (no EV).

\subsubsection{Workload}

The NASA TLX assessed workload using six scales associated with mental, physical, and temporal demand, performance, effort, and frustration level. The raw TLX scoring was used based on Hart and Staveland. ${ }^{5}$

An ANOVA was conducted on display condition for the dependent variable, NASA TLX (using the total of the 6 attribute ratings). Because the Mauchly's Test of Sphericity was significant, the Greenhouse-Geisser correction was applied, $F(18,119)=2.871, p<0.01$.

Because of the large number of testable conditions (19 including baseline condition), the pair-wise comparisons with Bonferroni correction did not yield significance (using a significance level $\alpha$ of $0.002=0.05 / 19$ ). For display condition, the objective was to determine whether there were any significant differences for all display conditions (type, parallax, minification) as a whole compared to the baseline condition. Therefore, a planned comparison was conducted for each display condition compared to the baseline condition. The planned comparisons showed that none of the conditions were significantly different in perceived mental workload qualitative ratings from the baseline with the exception of the following:

1. $90^{\circ} \mathrm{AD}$, Minified FOR (M), Large Parallax, $F(1,19)=4.860, p=0.041$

The result confirms that the $90^{\circ} \mathrm{AD}$ location presents workload issues to the pilot particularly when the display has a large minification and large parallax. Although the other $90^{\circ}$ locations were not found to be rated significantly lower for workload than baseline, the descriptive results do suggest that pilots tended to consider the $90^{\circ}$ location poorer for workload compared to baseline.

\subsubsection{Post-run questions}

Nine questions were presented to crews and asked for their level of agreement on a 7-point Likert scale:

1. I was able to maintain taxi accuracy during SMGCS operation.

2. I was aware of ownship position on the airport surface.

3. I was aware of the cleared SMGCS taxi route.

4. I was aware of traffic and other vehicles during SMGCS operation.

5. I was aware of SMGCS signage, markings, and visual aid.

6. The FLIR presentation was effective for SMGCS taxi operation (ease of access, size, etc.). 
7. The display concepts and SMGCS charts contributed to communication effectiveness (ATC and Flight Crew)

8. The display concepts and SMGCS charts promoted crew resource management, coordination, and cohesion.

9. The display concepts and SMGCS charts contributed to perceived safety during SMGCS taxi operation.

The analysis of the post-run questionnaire data for display condition show some advantages of EV presentation only for Question 1 ("I was able to maintain taxi accuracy during SMGCS operations") and Question 2 ("I was aware of ownship position on the airport surface"). Pilots reported being able to better maintain taxi accuracy during SMGCS operations for most display conditions, except HUD, compared to baseline; those non-HUD displays that were non-significant were due largely because of minification factor. At the visibility condition tested, there were sufficient cues OTW for tactical vehicle control. Since the EV cues on the HUD did not align perfectly with the OTW cues due to parallax, some crews found this distracting. Flight crews also reported being significantly more aware of ownship position on the airport surface with the FLIR except in the $90^{\circ} \mathrm{AD}$ location with minified and large parallax condition. The other post-run question data did not show significant differences; the EV display concepts were no different than the baseline (no EV) condition.

Overall, for the EV display conditions (18 experimental conditions), there were few practically significant effects when results were analyzed solely by display condition. Instead, the data were analyzed as a function of the experimental treatment manipulations which were: (a) effect of display type, (b) effect of minification, and (c) effect of parallax as described below.

\subsection{Effects of parallax}

\subsubsection{Average taxi speed}

An ANOVA was conducted on the average taxi speed for EV Display Location (HUD, MFD, Center AD, $60^{\circ} \mathrm{AD}$, $90^{\circ} \mathrm{AD}$ ) and parallax (Small, Large). The display FOV was fixed at Normal for this analysis. Levene's test for equality of variances was met for the present analysis. EV display location $(F(4,81)=0.143, p=0.966)$, parallax $(F(1,81)=1.2652, p=0.264)$, and the second-order interaction of the FLIR display location and parallax $(F(4,81)=0.126, p=0.973)$ were not significant for average taxi speed. Operationally, taxi speed was not influenced by the location or the amount of parallax in the EV. The overall mean taxi speed was 11.3 knots with a standard deviation of 2.4 knots for the normal display FOV runs.

\subsubsection{Head-up time, pilot flying}

An ANOVA was conducted on the PF head-up time percentage dependent variable for FLIR Display Location (HUD, MFD, Center AD, $60^{\circ} \mathrm{AD}, 90^{\circ} \mathrm{AD}$ ) and parallax (Small, Large). The display FOV was fixed at Normal for this analysis. Levene's test for equality of variances was met for the present analysis. EV display location $(F(4,71)=0.420, p=0.794)$, parallax $(F(1,71)=0.061, p=0.806)$, and the second-order interaction of FLIR display location and parallax $(F(4,71)=0.292, p=0.883)$ were not significant for PF head-up percentage time. Operationally, $\mathrm{PF}$ head-up percentage time was not influenced by the location or the amount of parallax in the EV. The overall mean PF head-up time percentage was $71.3 \%$ with a standard deviation of $12.7 \%$ for the normal display FOV runs.

\subsubsection{Head-up time, pilot monitoring}

An ANOVA was conducted on the PM head-up time percentage dependent variable for FLIR Display Location (HUD, MFD, Center AD, $60^{\circ} \mathrm{AD}, 90^{\circ} \mathrm{AD}$ ) and parallax (Small, Large). The display FOV was fixed at Normal for this analysis. Levene's test for equality of variances was met for the present analysis. EV display location $(F(4,78)=2.362, p=0.060)$, parallax $(F(4,78)=2.052, p=0.156)$, and the second-order interaction of EV display location and parallax $(F(4,78)=0.673, p=0.613)$ were not significant for PM head-up percentage time. Operationally, PM head-up percentage time was not influenced by the location or the amount of parallax in the EV. The overall mean PM head-up time percentage was $55.9 \%$ with a standard deviation of $14.7 \%$ for the normal display FOV runs. 


\subsubsection{Situation awareness}

No significant main effects or interactions $(p>0.05)$ were found for effect of parallax (Small, Large) on the SART. Flight crew ratings for SART were practically identical for the small parallax condition ( mean $=7.36$, std.dev. $=$ $3.029)$ compared to large parallax condition $($ mean $=7.48$, std.dev. $=3.002)$.

\subsubsection{Workload}

No significant effects were found for NASA-TLX for effect of parallax.

\subsubsection{Post-run questions}

For parallax effects, there were significant findings:

- Run Questionnaire Item \#1, $p=0.009$

"Able to maintain taxi accuracy during SMGCS operations"

- Run Questionnaire Item \#5, $p=0.002$

"Aware of SMGCS signage, markings, and visual aids"

- Run Questionnaire Item \#7, $p=0.044$

"Display concepts and SMGCS charts contribute to communication effectiveness"

- Run Questionnaire Item \#8, $p=0.014$

"Display concepts and SMGCS charts promoted CRM"

- Run Questionnaire Item $\# 9, p=0.010$

"Display concepts and SMGCS charts contribute to perceived safety"

The results suggest that flight crews preferred large parallax view angles for maintaining taxi awareness and accuracy; awareness of SMGCS signage, markings, and visual aids; communication effectiveness; and coordination; and enhanced safety. However, the numerical differences indicate that these differences do not represent an operational or practical significant difference.

\subsection{Effects of minification}

\subsubsection{Average Taxi Speed}

An ANOVA was conducted on the average taxi speed dependent variable for EV Display Location (HUD, MFD, Center $\mathrm{AD}, 60^{\circ} \mathrm{AD}, 90^{\circ} \mathrm{AD}$ ), Display FOV (Normal, Minified), and parallax (Small, Large). The HUD runs were excluded from this analysis since they only have one FOV level (i.e., normal FOV). EV Display Location $(F(3,137)=0.113, p=0.952)$, Display FOV $(F(1,137)=0.611, p=0.436)$ and parallax $(F(1,137)=0.448, p=$ $0.504)$ were not significant for average taxi speed. None of the second-order interactions between the main factors were significant $(p>0.05)$ for this measure. The overall mean taxi speed was 11.4 knots with a standard deviation of 2.3 knots for these runs.

\subsubsection{Head-up time, pilot flying}

An ANOVA was conducted on the PF head-up time percentage dependent variable for EV Display Location (HUD, MFD, Center AD, $60^{\circ} \mathrm{AD}, 90^{\circ} \mathrm{AD}$ ), Display FOV (Normal, Minified), and parallax (Small, Large). The HUD runs were excluded from this analysis since they only have one FOV level (i.e., normal FOV). The secondorder interaction between EV Display Location and parallax $(F(3,131)=3.159, p=0.027)$ was significant for head-up time percentage. The PF spent more time head-down when there was a large parallax error compared to the small parallax error when using the off-axis (60 and $90 \mathrm{deg}$ ) auxiliary displays and the head-down MFD. This trend was not true when using the center auxiliary display, where pilots spent more head-down time with the small parallax FLIR. Operationally, these trends may not be relevant since the largest difference between head-up time for the large and small parallax values was only $8 \%$ and occurred with the center auxiliary display which (incidentally) was located in a more head-up position than the other 3 displays. The main factors, EV 
Display Location $(F(3,131)=0.259, p=0.855)$, Display FOV $(F(1,131)=2.804, p=0.096)$ and parallax $(F(1,143)=0.355, p=0.552)$ were not significant for this measure. The remaining second-order interactions were also not significant $(p>0.05)$ for head-up time percentage. The overall mean PF head-up time percentage was $69.8 \%$ with a standard deviation of $12.0 \%$.

\subsubsection{Head-up time, pilot monitoring}

An ANOVA was conducted on the PM head-up time percentage dependent variable for EV Display Location (HUD, MFD, Center AD, $60^{\circ} \mathrm{AD}, 90^{\circ} \mathrm{AD}$ ), Display FOV (Normal, Minified), and parallax (Small, Large). The HUD runs were excluded from this analysis since they only have one FOV level (i.e., normal FOV). The main factors, EV Display Location $(F(3,133)=0.819, p=0.486)$, Display FOV $(F(1,133)=0.377, p=0.540)$ and parallax $(F(1,133)=0.058, p=0.810)$ were not significant for this measure. None of the second-order interactions between the main factors were significant $(p>0.05)$ for this measure. Operationally, no differences were seen with PM head-up time percentage due to the EV display location, parallax, or display FOV. The overall mean PM head-up time percentage was $55.1 \%$ with a standard deviation of $15.4 \%$.

\subsubsection{Situation awareness}

No significant main effects or interactions $(p>0.05)$ were found for effect of minification (Normal, Minified) on the dependent variable of SART.

\subsubsection{Workload}

No significant main effects or interactions were found for effect of minification.

\subsubsection{Post-run questions}

No significant effects were found for effect of minification on the post-run questionnaires.

\subsection{Off-nominal conflict detection}

Two display types (HUD, Center AD) were evaluated for detection of off-nominal events during low visibility surface operations. The off-nominal scenario involved a situation where a baggage cart was inadvertently left on a taxiway. The location was also right after a cleared turn onto the taxiway and the hold short of the active runway for departure. As it was off-axis during the turn, the baggage cart was not visible out the window during the turn and it was not illuminated by the nose-mounted landing light. The cart was, however, visible in the field-of-view of the EV imagery well prior to the turn for both display conditions tested. Therefore, the off-nominal was designed to evaluate the vigilance of the flight crew to monitor FLIR imagery, as well as the outside view in SMGCS/LVO, and the potential for the EV to augment hazard avoidance.

The display conditions tested were: HUD (non-minified, small parallax) and Center AD (non-minified, small parallax). The display conditions were chosen to control for effect of head-up versus head-down and minimize issues of off-axis presentations (i.e., the $60^{\circ} \mathrm{AD}$ and $90^{\circ} \mathrm{AD}$ display conditions). The display conditions differ only in that the EV was either displayed on the HUD or the Center AD.

No crew hit the baggage cart. Minimum detection distances varied from 51 feet to 124 feet. Detection of the hazard by crew was 30\% PF, 40\% PM, and 30\% both. Detection of the hazard by type was $70 \%$ OTW, $20 \%$ $\mathrm{EV}$, and $10 \%$ both.

\subsection{Post-experiment questionnaire}

A semi-structured verbal debrief session was held after the post-experiment questionnaire was complete. This session generally lasted between 30 and 45 minutes and included items commented upon in the questionnaires, additional issues the pilots noticed during the runs, specific items the researchers had noticed during that particular crew's scenarios, and general comments concerning LVO/SMGCS, and this experiment.

In one question, crews were asked to rate the fidelity of the simulation to real-world SMGCS operations on a scale from 1 to 7 ( 1 completely unrealistic and 7 being completely realistic). The average rating of the simulation fidelity was 6 . 


\subsection{Simulation Sickness Questionnaire}

Of the 120 SSQs given (6 SSQs per pilot, 12 per crew and 10 crews total), 31 (26\%) of those questionnaires had at least one symptom reported. A total of 67 symptoms were reported in all and $70 \%$ of those were by the PF. One PF reported 29 (62\% of all pilots flying) symptoms. If you remove this PFs data as an outlier, then the reported symptoms were almost evenly split between the PFs and PMs.

One PF reported having moderate fatigue throughout the day. One PM reported moderate eye strain. Other than the reported symptoms above, all other symptoms were marked as being slight. Two crews (20\%) did not report any simulation sickness symptoms on any of their questionnaires. The most common symptom reported by crews was slight fatigue which was reported by $70 \%$ of the crews at some point during the day. Usually, the reporting of fatigue occurred before the end of a data block before a break (scheduled break, lunch, end of data collection).

One PM reported stomach awareness (a precursor to nausea) from viewing the $90^{\circ} \mathrm{AD}$. This $\mathrm{PM}$ noted the stomach awareness when looking at video that was not aligned with the aircraft.

\section{DISCUSSION OF RESULTS}

A simulation of low visibility surface operations were conducted for various arrival and departure scenarios. Crews felt the simulation of SMGCS operations was realistic in terms of simulated weather conditions and workload. Most crews reported no differences in their company's Standard Operating Procedures (SOPs).

For all but the $90^{\circ} \mathrm{AD}$ condition, the display of EV was not measurably different than the baseline, no EV condition for taxi speed, head-up time, workload, or situation awareness. Situation awareness and workload was rated worse for the $90^{\circ} \mathrm{AD}$ condition than the baseline. The $90^{\circ} \mathrm{AD}$ was the least preferred $\mathrm{EV}$ display by most crews as the display was not in the primary nor secondary FOV. Pilots would have to turn their head and actively look for the display creating significant head-down time as compared to glancing at the other EV locations. It was also noted that having the $90^{\circ} \mathrm{AD}$ so far off boresight could have the potential to create motion sickness. This motion sickness was due to the FLIR video showing motion not inline with the motion of the aircraft.

At the visibility conditions tested, there were sufficient cues OTW for tactical vehicle control. Generally, crews used the EV display as a confirmation tool rather than a primary tool for taxi route awareness. Though lights tend to be identified earlier, taxiway signs were not discernible in the EV image. Taxiway signs are used by crew as a confirmation of SA and the signs were only visible OTW. Since the EV cues on the HUD did not align perfectly with the OTW cues due to parallax, some crews found this distracting and detracted from the PF's ability to use the EV image on the HUD as a confirmation tool.

The parallax in the EV image created a ground rush effect especially for the large parallax with the FLIR camera being much closer to the ground than the pilot's eye. Crews commented that this parallax gave the perception of a higher taxi speed.

Seeing the clearance bar lights of the Geographic Position Markers (GPMs) in the EV image was valuable in predicting when to start searching for the GPM. Since the EV sensor parallax was simulated to be lower than the pilot's eye, the view angle of the airport surface was lower than the OTW view. Due to this parallax, the GPM is not in view of the EV image very long, approximately 1 second or less depending on ground speed. Essentially, seeing the clearance bar lights early reduced workload as the PM had a better prediction of when the GPM would be visible. Thus, crews used the clearance bar lights to anticipate upcoming GPM for position confirmation.

Crews stated that the EV image either enhanced or had no effect on Crew Resource Management (CRM). None of the crews felt the EV display was a negative impact on overall CRM. 


\section{CONCLUSIONS}

Twenty pilots (10 crews) participated in a motion-base simulation experiment using real-world operational procedures and communication, with external air and surface traffic during LVO/SMGCS operations. The goal of the research was to evaluate the use of EV during LVO/SMGCS and assess the impact of different EV flight deck design considerations, specifically, display location, image minification, and camera parallax.

The experimental used $5 \mathrm{EV}$ display locations, $2 \mathrm{EV}$ fields-of-regard, and 2 parallax error conditions, in partially factorial within-subjects design. A baseline condition without EV was also evaluated for comparative purposes. Departure and arrival scenarios were flown as well as one unexpected, emulating SMGCS LVO night 500 RVR operations at Memphis International Airport with the required SMGCS visual aids and procedures specified in the Memphis SMGCS plan. The EV (i.e., FLIR) simulation was calibrated to show topographical objects within a range of approximately $600 \mathrm{ft}$ and light sources within a range of approximately $1000 \mathrm{ft}$.

With the OTW visual cues, there were sufficient cues OTW for tactical vehicle control. As such, EV was used as conformation tool and for all but the $90^{\circ} \mathrm{AD}$ condition, the display of $\mathrm{EV}$ did not measurably impact the operation. There were no significant differences between the EV experimental conditions and the baseline, no EV condition on taxi speed, head-up time, workload, or situation awareness. The display of EV, 90 deg outboard of the flight crew did cause degradations in the workload, SA, and acceptability. The display of EV on the HUD was sometimes distracting, because by design, the EV was not perfectly aligned with the OTW. Although EV did not measurably improve performance during surface operations, EV was found to be subjectively beneficial, especially to the PM, in terms of enhanced monitoring and improve situation awareness.

Future work includes evaluating the use of EV when certain required LVO/SMGCS enhanced visual aids are not present. Further, only one EV and one OTW natural visibility condition was tested. The interaction between OTW and enhanced visibility should be evaluated including Level 1 and Level 2 LVO/SMGCS conditions. Finally, the integration of Airport Moving Map (AMM)concepts with LVO/SMGCS chart symbology and EV usage should be tested. AMMs allow the crew to quickly identify their position on the airport surface greatly increasing their SA for surface operations. It is believed that coupling this technology with an electronic SMGCS presentation may provide an enhancement during low visibility surface operations; however, the effect on pilot workload and head-down time must be assessed.

\section{ACKNOWLEDGMENTS}

This work was jointly sponsored by NASA's Aviation Safety Program (AvSP), Vehicle Systems Safety Technologies project (Mr. Paul Krasa, Program Manager, Mr. Randall Bailey, Project Scientist) and the FAA Human Factors R\&D Project for NextGen, led by Dr. Tom McCloy and Mr. Stephen Plishka. The authors gratefully acknowledge the contributions of Terry King (FAA) and many NASA Langley technicians, programmers, and engineers who support/have supported these research projects. Notable among them are Regina Tober, Lon Kelly, Ming Shih, Jerry Karwac, Wei Anderson, Thomas Feigh, Chris Harrison, Victoria Chung, Sean Kenny, Dennis Frasca, Tom Wolters, Joe Whiting, Wayne Burge, Catherine Buttrill, Kemper Kibler, Darrell Sacra, Philip Smith, Sherri Rehfeld, Sonia Herndon, Dale Ashcom, Ben Lewis, Brian Hutchinson, Lindsey Lowe, Donald Buhl, Steve Velotas, and Lisa Rippy.

\section{REFERENCES}

1. FAA, "Enhanced flight vision systems," Advisory Circular 90-106, US Department of Transportation, Federal Aviation Administration, Washington DC, USA, June 2010.

2. FAA, "FAA's NextGen implementation plan," tech. rep., US Department of Transportation, Federal Aviation Administration, Washington DC, USA, March 2011.

3. A. Alexander, L. Prinzel, C. Wickens, L. Kramer, J. Arthur, and R. Bailey, "Evaluating the effects of dimensionality in advanced avionic display concepts for synthetic vision systems," International Journal of Aviation Psychology 19(2), pp. 105-130, 2009.

4. RTCA, "Minimum aviation system performance standards (MASPS) for enhanced vision systems synthetic vision systems, combined vision systems and enhanced flight vision systems," Tech. Rep. DO-315, RTCA, Inc., December 2008. 
5. S. G. Hart and L. E. Staveland, "Development of a multi-dimensional workload rating scale: Results of empirical and theoretical research," in Human mental workload, P. A. Hancock and N. Meshkati, eds., pp. 139183, Elsevier, (Amsterdam, The Netherlands), 1988.

6. R. M. Taylor, "Situational awareness rating technique (SART): The development of a tool for aircrew systems design," in AGARD Conference Proceedings No 478, Situational Awareness in Aerospace Operations, pp. 3-1 - 3-37, (Aerospace Medical Panel Symposium, Copenhagen), October 1990.

7. R. S. Kennedy, N. E. Lane, K. S. Berbaum, and M. G. Lilienthal, "Simulator sickness questionnaire: An enhanced method for quantifying simulator sickness," International Journal of Aviation Psychology , pp. 203$220,1993$.

8. G. Dallal, "Confidence intervals involving logarithmically transformed data," May 2012. http://www.jerrydallal.com/LHSP/ci_logs.htm. 